\title{
Psychiatric and Psychological Research on Esports Is Not Limited to Professional Players
}

\author{
Veli-Matti Karhulahti \\ University of Jyväskylä, vmmkar@utu.fi
}

This is a response to a recently published letter by Griffiths (2020). That letter was written to correct and comment on an earlier report (Sharma et al. 2018), which concerned esports cyberbullying in India. Alas, Griffiths' letter only confuses the research field more. I aim to clarify those confusions here.

In the original report by Sharma and colleagues (2018), the scholars describe a case of a 19-year-old esports gamer who has played "multiplayer online battle arena games [MOBAs] from the age of 15 years" (16). Griffiths (2020) claims that the scholars were misusing the word 'esports player' because, with reference to his own work (Bányai et al., 2018), there is "general consensus that esports relates to professional videogame playing [therefore the] gamer described in Sharma et al.'s case study was not an esports player" (Griffiths 2020, 214). But there is no such consensus - not even in Griffiths' own studies. For sure, global professional scenes drive esports (e.g. Taylor, 2012; Scholz, 2019), but not all esports players are professionals. In fact, his own work (Bányai et al., 2018) explicitly states that no consensus on 
applying the term 'esports' in research exists; there are "different definitions of what esports comprise although there are some similar characteristics" (352). None of these characteristics that lead the field of esports research entail professional status from all players. To clarify still: one does not need to be a professional, by any of the many definitions, to play esports or other sports. Likewise, psychiatric and psychological research on esports - like psychiatric and psychological research on sports - is not limited to professional esports players.

Actually, the fact that Sharma and colleagues report the individual to have played the so-called MOBA genre - arguably the most popular type of esports today - indicates that there was nothing wrong with the terminology of their report. Had the described individual played a single-player or non-competitively inclined videogame genre, a reference to esports might have been misleading.

Griffiths continues: "The fact that videogame addicts and esports players are arguably behaviourally very similar if not identical ... does not mean they should be classed similarly." I agree that scholars should not loosely employ - or worse, confuse - labels for gaming such as 'esports' and 'addiction'. This, I would add, includes acknowledging a nonbinary reality with multiple esports skill levels and various degrees (and kinds) of possible gaming problems. In these domains, 'professionalism' and 'addiction' may be summoned to represent some complicated extremes. The complexity of this reality is the very reason why researchers generate hypotheses and collect evidence: with such efforts, we may, one day, be able to distinguish between challenging concepts like the above in meaningful ways. Many have shown that the currently dominant methods for studying gaming-related 'addiction' and its survey-based screening need much more work (Przybylski et al. 2016; Ferguson et al. 2019) - and the critical resources of leading scholars should be focused on solving these issues, rather than claiming consensus. 
Griffiths' claim that playing esports should be understood synonymously with 'professional' play guides him to one more false claim; namely, that "there is no evidence in the empirical literature that cyberbullying takes place within esports video gaming [because] esports tournaments are played in front of huge audiences." While 'cyberbullying' is another contested term that the present space does not allow probing further, there is plenty of evidence that esports games involve cyberbullying toxic behaviors in various forms on virtually all skill levels (see Kwak \& Blackburn 2014). Griffiths evidences his claim by reasoning that "cyberbullying by esports players would also be detrimental to their professional reputation" (Griffiths 2020, 214). Many esports professionals have, indeed, a bad reputation due to cyberbullying and toxicity and this is why the governing esport companies regularly punish them. One could explore, for instance, the open punishment history of professional players by the esports company in this introductory Nature article (Maher 2016).

Griffiths ends by wondering why his work was not cited: "Sharma and colleagues [appear to claim] that cyberbullying within online gaming is a new area but I and my colleagues have been publishing papers on this phenomenon since $2012 \ldots$ yet no reference was made" (Griffiths 2020, 214). Griffiths, who does not fail to self-cite those publications, must well know that the research of this popular topic begun proliferating in the early 1990s - for which it is unclear why Sharma and colleagues should cite Griffiths' recent work. I conclude that Sharma and colleagues (2018) did not need to be corrected about the issues pointed out by Griffiths. In case any of the above need further clarification, I spend the $10^{\text {th }}$ and last allowed reference to my monograph (Anonymous 2020), which explains all of those issues in more detail and cites relevant literature. 


\section{References}

Anonymous, 2020.

Bányai, F., Griffiths, M.D., Király, O., Demetrovics, Z., 2018. The psychology of esports: a systematic literature review. J. Gambl. Stud. http://dx.doi.org/10.1007/s10899-018- 9763-1

Ferguson, C. J., Bean, A. M., Nielsen, R. K., \& Smyth, M. P., 2019. Policy on unreliable game addiction diagnoses puts the cart before the horse. Psychology of Popular Media Culture.

Griffiths, M., 2020. Esports should not be confused with video gaming when reporting cyberbullying. Asian J. Psychiatry. https://doi.org/10.1016/j.ajp.2018.04.008

Kwak, H., \& Blackburn, J., 2014. Linguistic analysis of toxic behavior in an online video game.

Proceedings of the International Conference on Social Informatics. Springer.

Maher, B., 2016. Can a Video Game Company Tame Toxic Behaviour? Nature, 531

(7596): 568-71.

Przybylski, A. K., Weinstein, N., \& Murayama, K., 2016. Internet gaming disorder: Investigating the clinical relevance of a new phenomenon. American J. Psychiatry, 174(3), 230-236.

Scholz, T., 2019. eSports is Business. Springer.

Sharma, M.K., Anand, N., Mathew, V., 2018. Case report on implication of E-sport for changing platform of expression of bullying. Asian J. Psychiatry.

http://dx.doi.org/10.1016/j.ajp.2018.03.019

Taylor, T., 2012. Raising the stakes: E-sports and the professionalization of computer gaming. MIT Press. 